\title{
Analysis of BGP Multihomed Link Utilization Effect on Internet Services (http, ftp and email)
}

\author{
M.T. Moubarak \\ Dept. of Communications, \\ Military Technical College \\ Cairo, Egypt
}

\author{
Ashraf Diaa Elbayoumy \\ Dept. of Communications, \\ Military Technical College \\ Cairo, Egypt
}

\author{
Ahmed A. Abd El-Hafez \\ Dept. of Communications, \\ Military Technical College \\ Cairo, Egypt
}

\author{
Mohamed H. Megahed \\ Dept. of Communications, \\ Military Technical College \\ Cairo, Egypt
}

\begin{abstract}
Different internet services and network performance represented in end-to-end delay and jitter are studied with respect to different link utilization. Internet users will access the internet services through simulated internet network contains number of BGP routers and Multihomed links. Both link utilization effect on the different internet services and the inability of BGP to automatically redistribute the traffic over available Multihomed Links will be highlighted.This paper also introduce a simple network design which is very close to the real internet environment and covers internet users section, ISPs section and enterprise section which represents offered internet services hoping that this model give a hand in the BGP and internet services researches.
\end{abstract}

\section{Keywords}

BGP; Multihomed; HTTP; FTP; Email; OPNET.

\section{INTRODUCTION}

The Internet is one of the greatest achievements of humanity. Within only 40 years from its creation, its impact is staggering. It has radically changed and continues to change our society, including how we communicate, work, learn, shop, and play. Despite its tremendous success, the Internet has some great problems and the biggest problem of the Internet architecture is the inability to accommodate innovation in its fundamental architectural aspects and therefore address long-standing design limitations [1].

Border Gateway Protocol (BGP) is the core routing protocol of the Internet. It maintains a table of IP networks or 'prefixes' which designate network reachability among autonomous systems (AS). It is described as a path vector protocol. BGP does not use traditional Interior Gateway Protocol (RIP, OSPF, ISIS ...) metrics, but makes routing decisions based on path, network policies and/or rule sets.The primary function of a BGP speaking system is to exchange network reachability information with other BGP systems. This network reachability information includes information on the list of Autonomous Systems (ASes) that reachability information traverses. This information is sufficient for constructing a graph of AS connectivity, from which routing loops may be pruned and, at the AS level, some policy decisions may be enforced [2].

Multihoming is a technique to increase the reliability of the Internet connection for an IP network using multiple links to the internet from different internet service providers. To allow redundancy and BGP routing used for traffic distribution over these multiple links [3-4].

The Internet performance could be affected due to overutilization or congestion of a certain path as well as DDOS attacks has a significant effect on link bandwidth. In such a case, internet applications will suffer from high delay which affects the quality of service or may lead to session failure between the source and destination.On this paper link utilization effect will be studied on the most used simple internet services HTTP, FTP and Email and show how the services performance has been degraded as the traffic load over link increased and this effect is significant specially when link is fully utilized or over utilized.

The limitation of the inter-domain routing system will be highlighted which is the most ossified component of the Internet and its inability to automatically redistribute the traffic over the different Multihomed links based on link utilization where network has been designed to use number of core routers connected through Multihomed links and BGP routing Protocol has been simply deployed over routers without adding any routing policy to highlight this problem.OPNET Modeler has been used to design ,implement and simulate the desired network for this analysis and obtain the different results [5-6].

In section II, network design and implementation has been described in details and each network section internet users, ISPs and enterprise has been fully identified and represented. In section III, attributes and measurements of network and services has been identified. In section IV, simulation results has been analyzed and network and services performance has been highlighted per each service. In section $\mathrm{V}$, conclusion has been represented and direction for problem solving has been mentioned. Finally in section VI, future work has been mentioned.

\section{NETWORK DESIGN AND IMPLEMENTATION}

Network simulation has been performed to allow end-toend connectivity where the traffic of different internet services will flows form internet user side to the enterprise servers through the internet and accordingly network is divided into three parts Users, internet service providers(ISP) and enterprise that has different 
basic services like FTP, HTTP and Email that users need to access.

Simulated network section described as follows:

a- Internet Users section consists of users grouped in different LANs where each LAN initiates different services traffic and LAN switch connected finally to Router that carries user traffic to ISP through number of BGP Multihomed links.

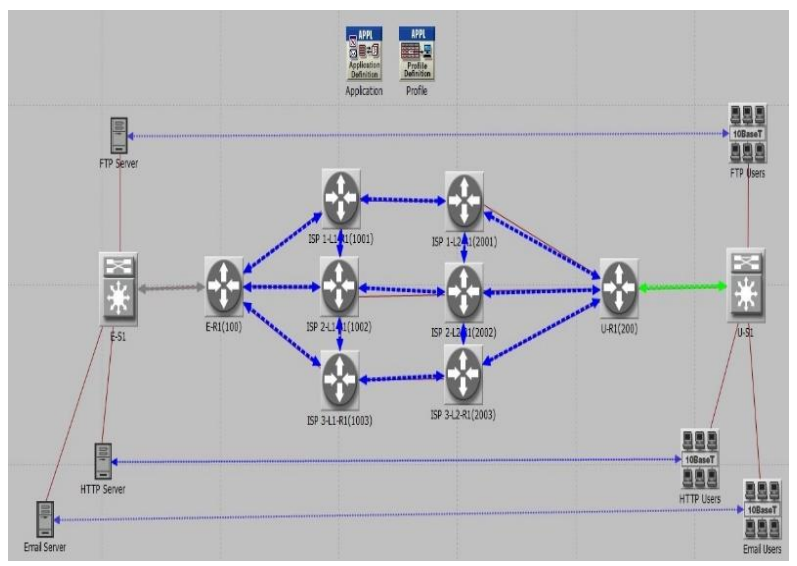

Fig. 1: Network Topology

b- ISP section which consists of number of ISPs each one is represented by a BGP router and number of Multihomed links which is connected to each other and two ISP levels are represented where ISP level one connected to enterprise section where level two connected to internet users section.

c- The two ISP levels has been used to make the network more realistic and matches the real internet environment

d- The enterprise section represents the service part where number of servers has been deployed to offer different services HTTP,FTP and Email to the internet users and all are connected together with LAN switch and then to the router which is connected to the internet via number of BGP Multihomed links. 10Mbps links have been used to connect servers to the enterprise LAN.

\section{MEASUREMENTS}

The Performance for the network and service will be measured, compared and then analyzed according to different traffic loads with 50\%,75\%, $100 \%$ and $120 \%$ percent of link capacity to test the effect of link utilization on the service performance.

- Network performance will be represented by an End-to-End delay and jitter.

- $\quad$ HTTP service performance will be measured by both page response time and object response time.

- FTP and Email services performance will be measured by both download response time and upload response time.

\section{PERFORMANCE ANALYSIS}

Enterprise has been connected to the internet through three Multihomed Links form different ISP and simple BGP configuration has been applied and each link utilization has been measured.According to figure 2 only one link has traffic and its utilization reaches $100 \%$ while other two links have no traffic and this proofs that BGP doesn't automatically redistribute traffic over available links and it can only support manual traffic distribution by applying routing polices and this partially solves the problem but doesn't solve the issue when link is fully or over utilized to move the some traffic to other links which has available bandwidth and it isn't utilized.

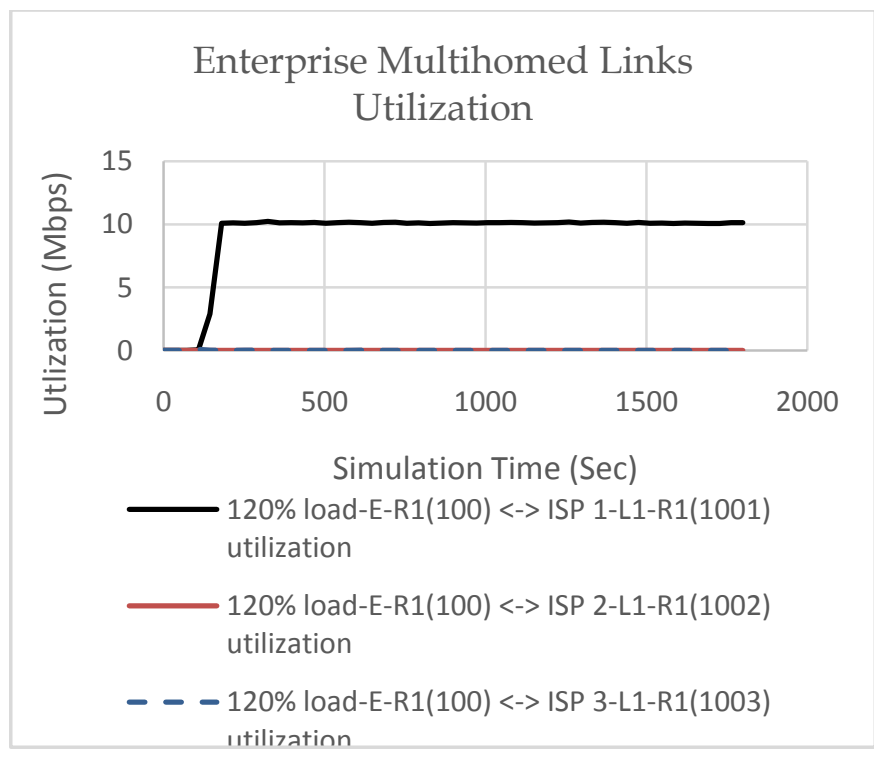

Fig. 2: Enterprise Multihomed Links Utilization

Network end-to-end delay [7] and Jitter have been measured according to the different link loads (50\%, $75 \% .100 \%$ and $120 \%$ ).

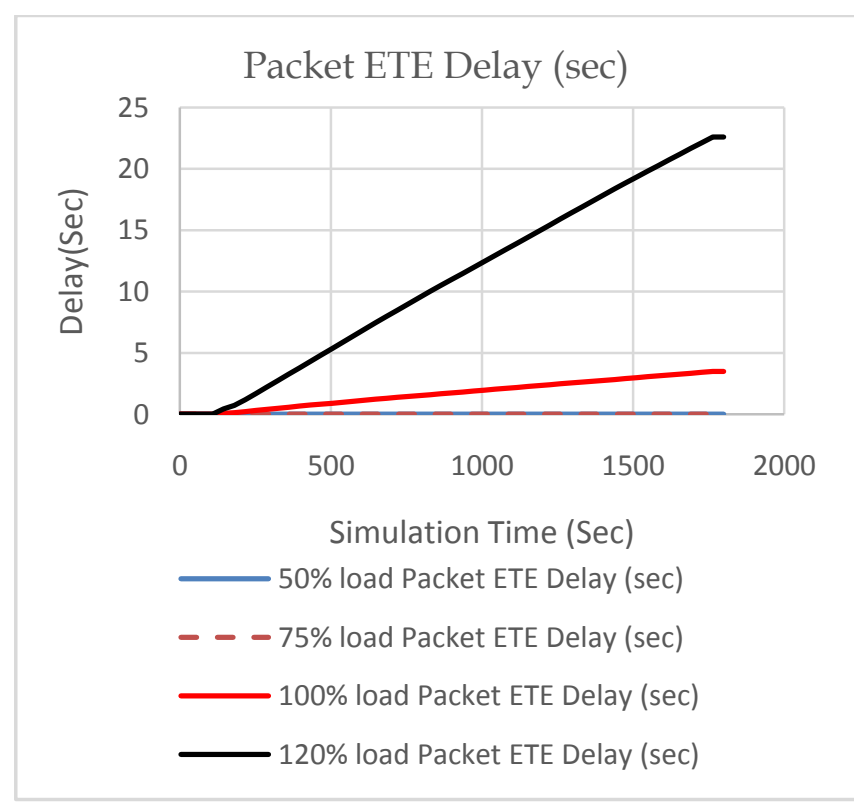

Fig. 3: Packet End-to-End Delay

Figure 3 shows that For link loads (50\% and $75 \%$ ) the delay varies from $1 \mathrm{msec}$ to $2 \mathrm{msec}$ and for (100\%) it increased to reach about $3.5 \mathrm{Sec}$ when link is fully utilized and finally 
when link has been over utilized with load (120\%) the delay significantly increased and reaches $22.5 \mathrm{Sec}$. which means that unacceptable service level has been reached.

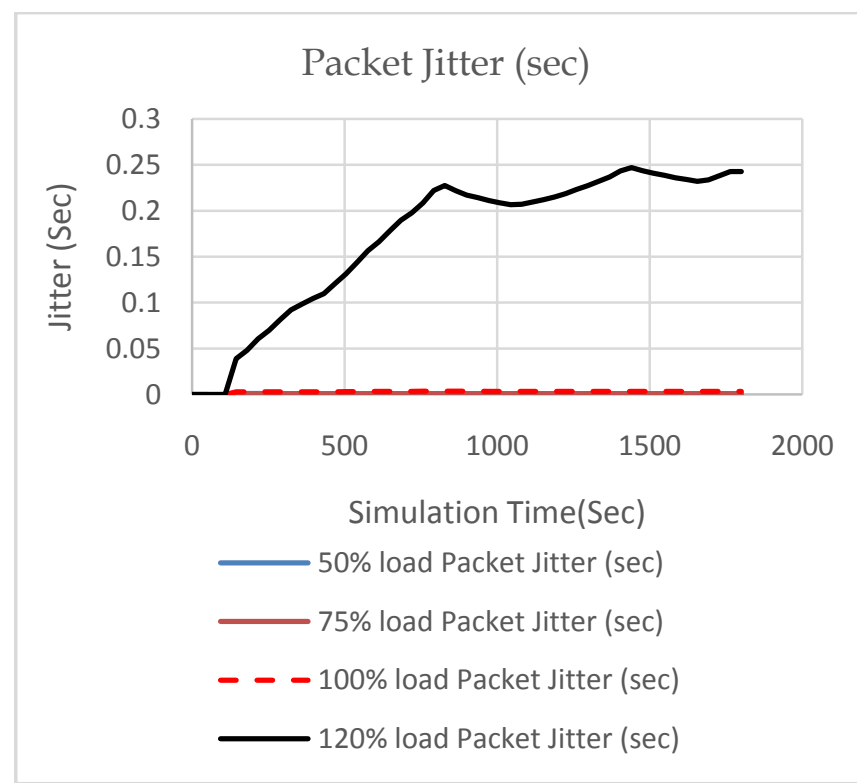

Fig. 4: Packet Jitter

Figure 4 shows that for loads $(50 \%, 75 \%$ and $100 \%)$ that jitter varies from about 0 to $3 \mathrm{msec}$ but when link is over utilized with (120\%) load it reaches to $246 \mathrm{msec}$.

Figure 5 shows http page response time which is defined as the time required to retrieve the entire page with all the contained inline objects.

For loads (50\% and 75\%) it varies from $440 \mathrm{msec}$ to 585 msec and for fully utilized link (100\%) load was $47 \mathrm{Sec}$ and then for over utilized link (120\%) load reaches 55.5 Sec.

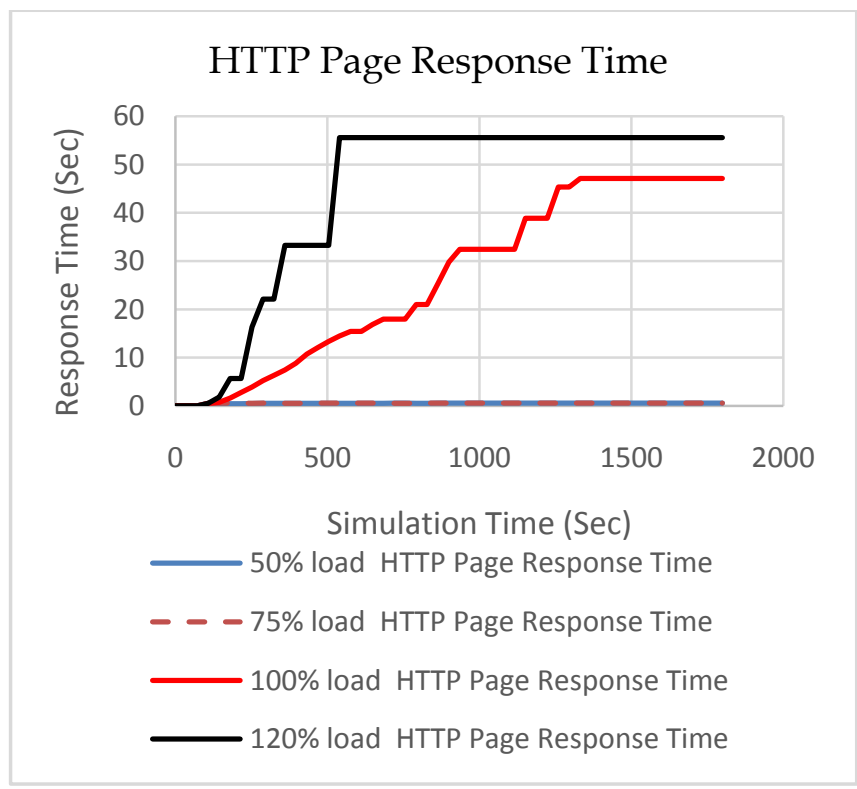

Fig. 5: HTTP Page Response Time

Figure 6 shows http object response time which is defined as response time for each inclined object from the HTML page.

For loads (50\% and 75\%) it varies from $93 \mathrm{msec}$ to 122 msec and for fully utilized link (100\%) load was $9.7 \mathrm{Sec}$ and then for over utilized link (120\%) load reaches 33.2 Sec.

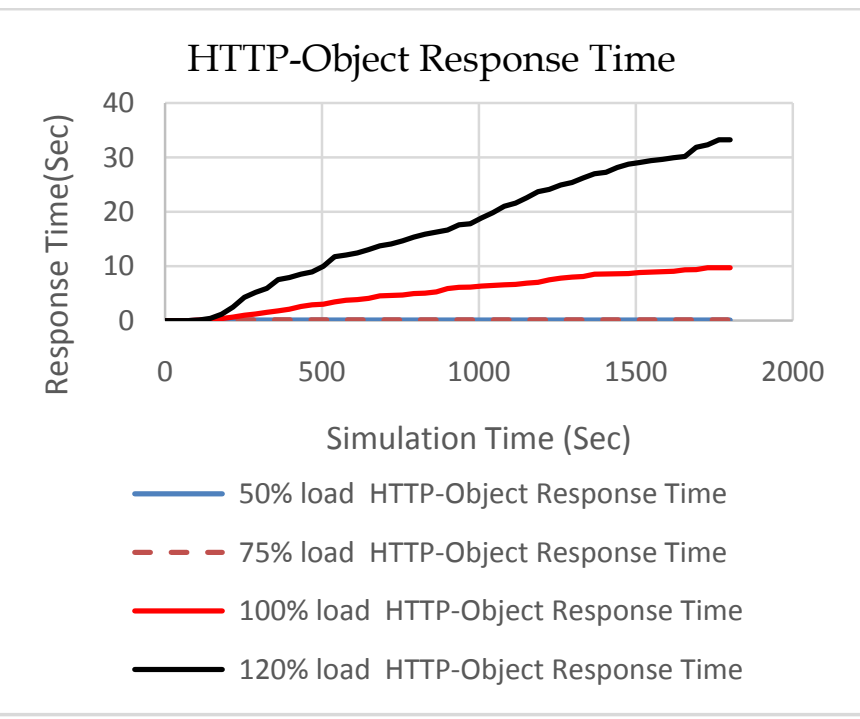

Fig. 6. HTTP Object Response Time

Figure 7 shows FTP download response time [8] which is defined as the time elapsed between sending a request and receiving the response packet.For loads (50\% and $75 \%$ ) it varies from $109 \mathrm{msec}$ to $126 \mathrm{msec}$ and for fully utilized link (100\%) load was $47 \mathrm{Sec}$ and then for over utilized link (120\%) load reaches 208 Sec.Figure 7 shows FTP download response time [8] which is defined as the time elapsed between sending a request and receiving the response packet.For loads $(50 \%$ and $75 \%)$ it varies from $109 \mathrm{msec}$ to $126 \mathrm{msec}$ and for fully utilized link $(100 \%)$ load was $47 \mathrm{Sec}$ and then for over utilized link (120\%) load reaches $208 \mathrm{Sec}$.

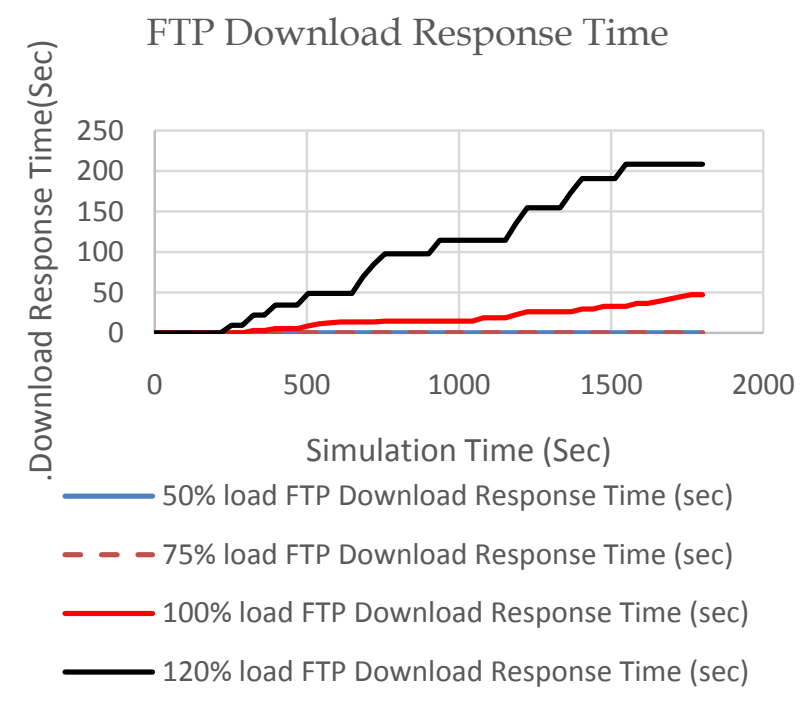

Fig. 2 : FTP Download Response Time

Figure 8 shows FTP upload response time [8] which is defined as the time elapsed between sending a file and receiving the response.For loads $(50 \%$ and $75 \%)$ it varies from $173 \mathrm{msec}$ to $276 \mathrm{msec}$ and for fully utilized link (100\%) load was $31.8 \mathrm{Sec}$ and then for over utilized link (120\%) load reaches $191 \mathrm{Sec}$. 


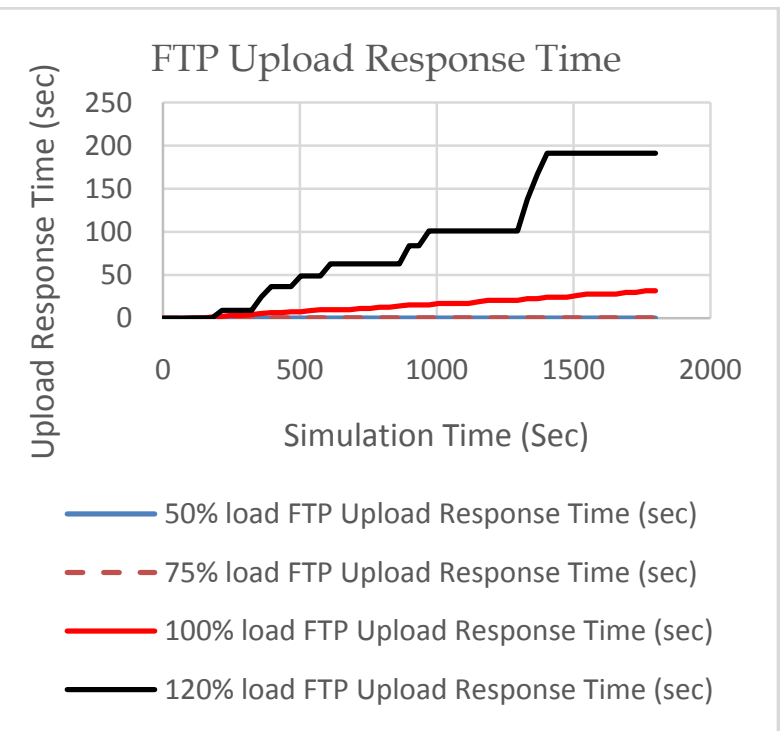

Fig. 3: FTP Upload Response Time

Figure 9 shows Email download response time [9] which is defined as the time elapsed between sending requests for emails and receiving emails from email server in the network.For loads .(50\% and $75 \%)$ it varies from $20 \mathrm{msec}$ to $24 \mathrm{msec}$ and for fully utilized link (100\%) load was 11 Sec and then for over utilized link (120\%) load reaches 120 Sec.

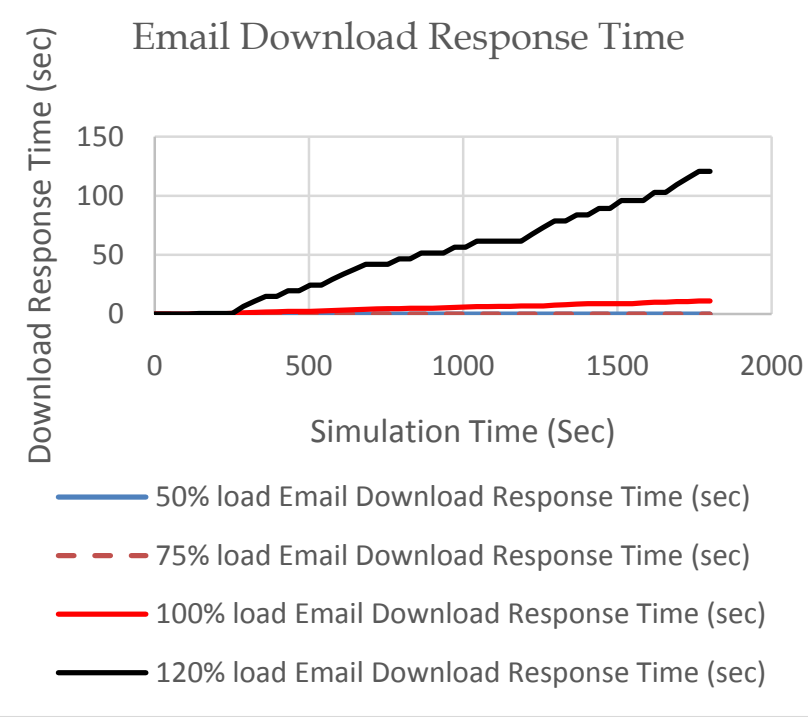

Fig. 4 : Email Download Response Time

Figure 10 shows Email upload response time [9] which is defined as the time elapsed between sending emails to the email server and receiving acknowledgments from the email server.For loads .(50\% and 75\%) it varies from 104 $\mathrm{msec}$ to $193 \mathrm{msec}$ and for fully utilized link (100\%) load was $13 \mathrm{Sec}$ and then for over utilized link (120\%) load reaches $134 \mathrm{Sec}$.

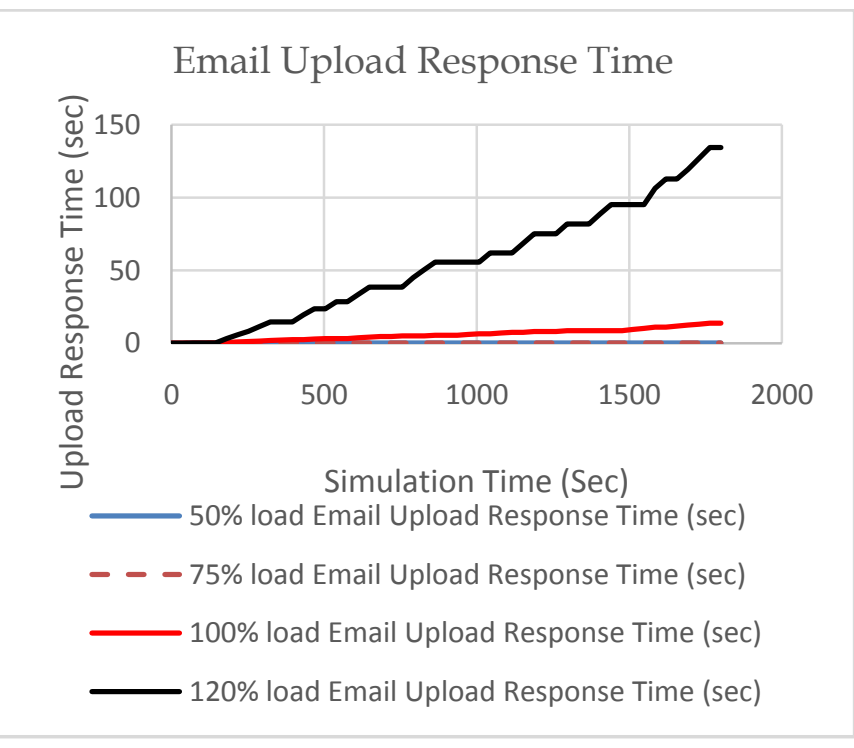

Fig. 5 : Email Upload Response Time

\section{CONCLUSION}

This paper presented a comparative analysis for selected internet services (HTTP, FTP and Email) to highlight two problems link utilization effect on internet services and the inability of the inter-domain routing system to automatically redistribute the traffic over available links based on link utilization. Link utilization has a great effect on internet offered services especially when link is fully utilized or over utilized and according to the above results the most affected service is FTP but HTTP users are more sensitive to the delay where when user downloading file or sending an Email just push the button to send and go to anything else while HTTP users write the required address and waiting for page to be loaded.

Enterprise and ISPs should maintain their internet links to be not fully utilized by using Multihomed links and apply BGP routing policies to manually distribute the traffic over the available links. The ossified inter-domain routing protocol (BGP) should be engaged with an intelligent mechanism to be able to fulfill and manage the internet scalability demands of bandwidth, services, applications, technologies and of course users.One of the key features that should be offered by the intelligent mechanism is to handle the internet traffic in smarter manner to automatically redistribute the traffic over the Multihomed connections based on link utilization.Software Defined Network (SDN) new networking paradigm in which the forwarding hardware is decoupled from control decisions. It promises to dramatically simplify network management and enable innovation.

\section{FUTURE WORK}

The future work will be developed to enhance the BGP routing algorithm to take into consideration the latency or link utilization in the calculation of route selection and then traffic redistribution based on latency or link utilization will be supported and accordingly the internet services performance will be enhanced as well as the interdomain routing protocol (BGP) be able to fulfill and manage the internet scalability demands of bandwidth, services, applications, technologies and of course users. 


\section{REFERENCES}

[1] European Research council (ERC) NetVolutionproject. http://netvolution.eu/scientific-approach.html

[2] Several RFCs define the BGP protocol, the following are the RFCs of interest to our research: RFC 4271, A Border Gateway Protocol 4 (BGP-4), RFC 4277, Experience with the BGP-4 Protocol, RFC 4276, BGP4 Implementation Report, RFC 4274, BGP-4 Protocol Analysis, RFC 4273, Definitions of Managed Objects for BGP-4, RFC 3392, Capabilities Advertisement with BGP-4, RFC 5065, Autonomous System Confederations for BGP, RFC 2918, Route Refresh Capability for BGP-4, RFC 4893, BGP Support for Four-octet AS Number Space, RFC 2439, BGP Route Flap Damping, RFC 4760, Multiprotocol Extensions for BGP-4

[3] Hiroshi Fujinoki, "Analysis on ideal network structures to improve reliability by multi-path and multi-homing BGP routing in the Internet", UltraModern Telecommunications \& Workshops, 2009. ICUMT '09. International Conference on Oct 2009.

[4] Hiroshi Fujinoki, "Improving Reliability for MultiHome Inbound Traffic: MHLB/I Packet-Level InterDomain Load-Balancing", Proceedings of the
International Conference on Availability, Reliability and Security, March 2009.

[5] J. Theunis, B. Van den Broeck, P. Leys, J. Potemans, E. Van Lil, A. Van de Capelle, OPNET in Advanced Networking Education, OPNETWORK 2002, 2002, Washington DC, USA.

[6] OPNET Technologies, Inc., The OPNET Simulator, http://www.opnet.com/

[7] S. Farhangi and S. Golmohammadi, "A Comparative study of IS-IS and IGRP protocols for real-time application based on OPNET," Advances in Electrical Engineering Systems 1.1, 2012, pp. 65-70.

[8] Sharma, Chitranjan, and Bhudev Tyagi. "Performance evaluation of TCP variants under different node speeds using OPNET simulator." Advance Computing Conference (IACC), 2013 IEEE 3rd International. IEEE, 2013.

[9] Kaur, Jagmeet, and Er Prabhdeep Singh. "SIMULATION BASED PERFORMANCE ANALYSIS OF IPV6 BASED IS_IS, OSPFV3 AND OSPFV3_IS-IS PROTOCOLS." (2014) 